\title{
No Effect of Levothyroxine and Levothyroxine-Induced Subclinical Thyrotoxicosis on the Pharmacokinetics of Sorafenib in Healthy Male Subjects
}

\author{
Funan Huang,, Antoinette Ajavon, Erya Huang,, John Lettieri, ${ }_{1}^{1}$ Rong Liu, ${ }_{1}^{1}$ Carol Peña, and Matthias Berse ${ }^{2}$
}

Background: Patients receiving the multikinase inhibitor sorafenib for locally recurrent or metastatic, progressive, differentiated thyroid carcinoma (DTC) refractory to radioactive iodine often receive concomitant levothyroxine for thyrotropin (TSH) suppression. In the Phase 3 DTC trial (DECISION), sorafenib exposure was approximately twofold higher than that observed in other cancers. This study assessed sorafenib pharmacokinetics without and with concomitant levothyroxine to examine whether a levothyroxine interaction or levothyroxine-induced subclinical thyrotoxicosis results in increased sorafenib exposure in patients with DTC. Methods: This was an open-label, two-period sequential treatment study in healthy male subjects. In period 1, day 1 , subjects received a single oral dose of sorafenib $400 \mathrm{mg}$, followed by a minimal 10-day washout. In period 2, day 1, levothyroxine $300 \mu \mathrm{g}$ was administered orally once daily (q.d.) for 14 days. After 10 days, a single oral concomitant dose of sorafenib $400 \mathrm{mg}$ was given. Blood samples for sorafenib pharmacokinetic analyses were obtained pre-dose and at time points up to 96 hours after sorafenib dosing. Samples for thyroid tests were collected before and after levothyroxine dosing.

Results: Twenty-five subjects completed the study and were evaluable for pharmacokinetic analysis. Levothyroxine $300 \mu \mathrm{g}$ q.d. was well tolerated and induced subclinical thyrotoxicosis, producing full suppression of $\mathrm{TSH}(M \pm S D=0.032 \pm 0.027 \mathrm{mIU} / \mathrm{L})$ and increased free thyroxine (from $0.94 \pm 0.09$ to $1.77 \pm 0.33 \mathrm{ng} / \mathrm{dL}$ ) and free triiodothyronine (from $2.87 \pm 0.28$ to $4.24 \pm 0.66 \mathrm{pg} / \mathrm{mL}$ ) levels by day 11 of period 2 . The geometric mean $(\% \mathrm{CV})$ sorafenib maximum concentration $\left(C_{\max }\right)$ without and with levothyroxine was 2.09 (68.1) and 1.78 (63.9) $\mathrm{mg} / \mathrm{L}$, respectively, with a corresponding geometric mean area under the curve of 68.1 (68.2) and 64.3 (66.3) $\mathrm{mg} \cdot \mathrm{h} / \mathrm{L}$. Median (range) time to $C_{\max }$ was $4.00(2.98-16.0)$ and 4.02 (1.98-36.0) hours, respectively. Mean (\%CV) half-life was 24.0 (25.3) and 25.7 (21.0) hours. All study drug-related adverse events were mild and included headache and fatigue for sorafenib, and headache, increased alanine aminotransferase and glutamate dehydrogenase, fatigue, and nervousness for levothyroxine.

Conclusions: Levothyroxine $300 \mu$ g q.d. for 14 days was well tolerated, induced subclinical thyrotoxicosis, and did not affect sorafenib pharmacokinetics. The findings suggest that concomitant use of levothyroxine with sorafenib is not likely responsible for the previously reported increase in sorafenib exposure in patients with DTC. However, the possible effects of long-term levothyroxine dosing were not assessed.

Keywords: thyroid cancer—clinical, clinical trials, clinical research

\section{Introduction}

$\mathbf{T}$

THE INCIDENCE OF THYROID CANCER is increasing at a faster rate than any other cancer in the United States
(1). The number of new cases has increased over the last 10 years at a rate of approximately $5 \%$ per year (2). In 2015 , thyroid cancer accounted for approximately $4 \%$ of all new U.S. cancer cases, making it the eighth most common

\footnotetext{
${ }^{1}$ Bayer HealthCare Pharmaceuticals, Whippany, New Jersey.

${ }^{2}$ CRS Clinical Research Services Berlin GmbH, Clinical Pharmacology Unit, Berlin, Germany.

(C) Funan Huang et al. 2017; Published by Mary Ann Liebert, Inc. This article is available under the Creative Commons License CC-BYNC (http://creativecommons.org/licenses/by-nc/4.0). This license permits non-commercial use, distribution and reproduction in any medium, provided the original work is properly cited. Permission only needs to be obtained for commercial use and can be done via RightsLink.
} 
cancer, with an estimated 62,450 new cases and 1950 deaths (2).

Histologic types of thyroid cancer include papillary (PTC; approximately $80 \%$ of all thyroid cancers), follicular (FTC; including conventional and oncocytic types; approximately $15 \%)$, poorly differentiated $(<5 \%)$, anaplastic $(1-2 \%)(3)$, and medullary (3-4\%) thyroid cancer (4). PTC and FTC are referred to as differentiated thyroid cancers (DTCs) (5). Although the majority of DTCs can be treated with surgery and radioactive iodine (RAI) therapy, a clinically significant proportion will ultimately develop into RAI-refractory DTC.

Sorafenib is a multikinase inhibitor that targets tumor cell proliferation and angiogenesis through inhibition of a number of kinases, such as vascular endothelial growth factor receptor-1, -2, and -3; platelet-derived growth factor receptors; oncogenes rearranged during transfection (RET; including $R E T / \mathrm{PTC}$ rearrangements); cKIT; and $R A F$ family members (including $\left.B R A F^{V 600 E}\right)(6-8)$. Sorafenib is indicated for the treatment of locally recurrent or metastatic progressive DTC refractory to RAI treatment $(9,10)$. In the pivotal Phase 3 DECISION study, sorafenib was shown to improve progression-free survival significantly compared to placebo in patients with locally advanced or metastatic progressive RAI-refractory DTC $(11,12)$. Population pharmacokinetic analysis showed that the mean steady-state exposure (area under the plasma concentration vs. time curve from 0 to 12 hours) of sorafenib in patients with DTC was approximately twofold higher when compared to patients with nonthyroid tumors in other sorafenib trials (DTC, $95.7 \mathrm{mg} \cdot \mathrm{h} / \mathrm{L}$; hepatocellular carcinoma $[\mathrm{HCC}], 48.9 \mathrm{mg} \cdot \mathrm{h} / \mathrm{L}$; and renal cell carcinoma [RCC], $41.1 \mathrm{mg} \cdot \mathrm{h} / \mathrm{L})(13)$.

The underlying reason for the increased sorafenib exposure is currently unknown. However, one hypothesis is that it could potentially be related to the concomitant use of levothyroxine in the DTC patient population, which is not commonly used in the HCC or RCC populations. Levothyroxine is a synthetic thyroid hormone that suppresses thyrotropin (TSH) when administered at supraphysiologic doses $(10,14)$, and TSH suppression is recommended in patients who have aggressive tumors to prevent the growth of thyroid follicular epithelial cells $(10,14)$. Levothyroxine was administered to patients with DTC in the DECISION study, but only to a small proportion of patients in the nonthyroid cancer clinical studies.

Levothyroxine has a wide drug-drug interaction spectrum, including inhibition of cytochrome P450 (CYP) 3A4 and UGT enzymes (UDP-glucuronosyltransferases), and it has been reported to increase the exposure of numerous drugs, including other tyrosine kinase inhibitors (15). Potential evidence of the effect of levothyroxine on CYP3A enzyme or UGT transporter activities in humans was demonstrated by Zhao et al. (16). Levothyroxine was shown to inhibit UGT1A1catalyzed 4-MU glucuronidation strongly in a dose-dependent manner, most likely by competitive inhibition. In addition, pharmacokinetic data reported by Takahashi et al. suggested that the thyroid hormone triiodothyronine (T3) co-administered with midazolam, a CYP3A substrate, significantly inhibited CYP3A enzyme activity and altered midazolam metabolism (17). Because sorafenib is metabolized by CYP3A4, this seemed like a plausible mechanism for a potential interaction, although a study in healthy volunteers failed to show any effect of co-administration of the strong CYP3A4 inhibitor ketoco- nazole on sorafenib pharmacokinetics (18). An alternate hypothesis was that the levothyroxine-induced thyrotoxicosis may influence sorafenib exposure.

The current study was conducted to determine whether levothyroxine is responsible for the increase in sorafenib exposure in patients with DTC by evaluating any potential pharmacokinetic and pharmacodynamic drug-drug interactions between sorafenib and levothyroxine in healthy male subjects. The study was conducted in healthy volunteers because a patient study would have required withholding of clinically indicated levothyroxine treatment after surgical resection.

\section{Materials and Methods}

\section{Subjects}

Subjects included in the study were healthy men aged 18 45 years with a body mass index (BMI) of $18.5-30.0 \mathrm{~kg} / \mathrm{m}^{2}$, body weight $\geq 65 \mathrm{~kg}$, and normal thyroid function, as indicated by thyroid examination, including total and free T3, total and free thyroxine (T4), TSH, anti-TSH receptor antibody, antithyroperoxidase antibody, antithyroglobulin antibody, and thyroid ultrasound.

Subjects were excluded who had a history of clinically significant metabolic, renal, hepatic, or central nervous system disorders, such as seizure, psychosis, and sleep disorders; a history of cardiovascular diseases, such as arrhythmia, hypertension, and ischemia; known or suspected cardiovascular disease, such as potential risk of atrioventricular block and arrhythmia; a history of ongoing thyroid disease; or if they had received iodine-containing contrast medium within two months before first study drug administration (9).

\section{Study design}

This was an open-label, nonrandomized, sequentialtreatment, Phase 1 study (NCT02332031). Subjects were screened within 28 days before administration of the first study drug. The study was performed in two sequential periods. A schematic of the study design is illustrated in Figure 1. In period 1, subjects received a single oral dose of sorafenib $400 \mathrm{mg}$ on day 1 . In period 2, levothyroxine $300 \mu \mathrm{g}$ was administered orally once daily (q.d.) on days 1-14. A second single oral dose of sorafenib $400 \mathrm{mg}$ was co-administered with levothyroxine $300 \mu \mathrm{g}$ on day 11 of period 2 after a washout period of $\geq 17$ days.

The protocol was reviewed and approved by the Ethikkommission des Landes Berlin (Ethics Committee of the State of Berlin) before the start of the study, and the study was conducted in accordance with the ethical principles of the Declaration of Helsinki and the International Conference on Harmonization guideline E6: Good Clinical Practice. All subjects provided written informed consent.

\section{Outcomes and assessments}

The primary objective of the study was to assess the effect of concomitant administration of levothyroxine on the pharmacokinetics of sorafenib. The secondary objective was to assess the safety of sorafenib administered without and with levothyroxine. 


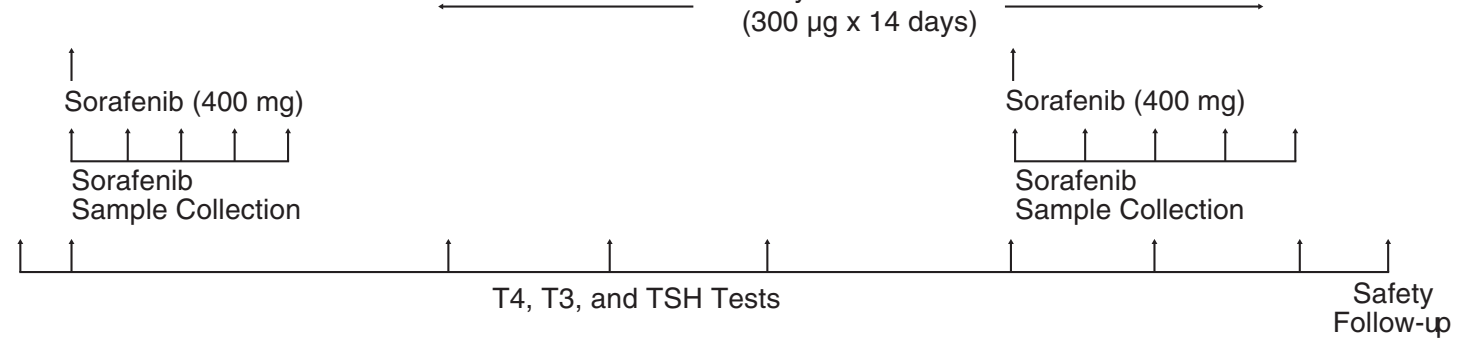

FIG. 1. Study design. S, screening; D, day; T3, triiodothyronine; T4, thyroxine; TSH, thyrotropin.

\section{Pharmacodynamic analysis}

Blood samples for determination of TSH and total and free $\mathrm{T} 3$ and $\mathrm{T} 4$ were obtained at the following time points: at screening, before sorafenib dosing in period 1 , and after levothyroxine dosing in period 2 (Fig. 1).

\section{Pharmacokinetic analysis}

Blood samples for pharmacokinetic analysis of sorafenib and its metabolite M-2 were collected at pre-dose and 1, 2, 3, $4,6,8,12,16,24,36,48,72$, and 96 hours after administration of sorafenib in periods 1 and 2. All pharmacokinetic parameters were calculated by the noncompartmental approach using the pharmacokinetic software WinNonlin ${ }^{\circledR}$ v5.3 (Certara, Princeton, NJ) in conjunction with the Automation Extension (WinAE v2.90; Bayer Pharma AG, Berlin, Germany). The main pharmacokinetic parameter calculated in this study was area under the plasma concentration versus time curve $(\mathrm{AUC})$ from time 0 to infinity $\left(\mathrm{AUC}_{0-\text { inf }}\right)$ after a single dose of sorafenib. Additionally, the following pharmacokinetic parameters were calculated for sorafenib: AUC from time 0 to the last data point greater than the lower limit of quantification (LLOQ; $\mathrm{AUC}_{0-\text { tlast }}$ ), maximum observed drug concentration in plasma after a single dose administration $\left(C_{\max }\right)$, half-life associated with the terminal slope $\left(t_{1 / 2}\right)$, time to reach $C_{\max }\left(t_{\max }\right)$, and AUC from time 0 to 96 hours $\left(\mathrm{AUC}_{0-96}\right)$. For sorafenib metabolite $\mathrm{M}-2$, the following pharmacokinetic parameters were calculated: $C_{\max }, \mathrm{AUC}_{0-\text { tlast }}$, $\mathrm{AUC}_{0 \text {-inf }}, t_{\max }$, and $t_{1 / 2}$.

Sorafenib and its metabolite M-2 were analyzed using reverse-phase liquid chromatography followed by tandem mass spectrometric detection. The method validation and analysis of the study samples were performed in compliance with the U.S. Food and Drug Administration Guidance on Bioanalytical Method Validation (2001) (19) and the European Medicines Agency Guideline on Bioanalytical Method Validation (2011) (20).

The calibration range for sorafenib was from 10.19 (LLOQ) to $10,386.08 \mu \mathrm{g} / \mathrm{L}$ (upper limit of quantification [ULOQ]). Mean inter-assay accuracy of back-calculated concentrations (except LLOQ) in calibrators ranged between $98.1 \%$ and $101.8 \%$, and precision was $\leq 9.0 \%$. Accuracy and precision at the lowest calibrator (LLOQ) were equal to $100.3 \%$ and $8.7 \%$, respectively. Quality control samples in the concentration range from 30 to $7219 \mu \mathrm{g} / \mathrm{L}$ were determined with an accuracy of $96.2-100.1 \%$ and a precision of $5.4-9.2 \%$.

The calibration range for metabolite M-2 was from 10.19 (LLOQ) to $2596.08 \mu \mathrm{g} / \mathrm{L}$ (ULOQ). Mean inter-assay accuracy of back-calculated concentrations (except LLOQ) in calibrators ranged between $98.1 \%$ and $104.7 \%$, and precision was $\leq 6.6 \%$. Accuracy and precision at the lowest calibrator (LLOQ) were equal to $99.9 \%$ and $7.9 \%$, respectively. Quality control samples in the concentration range 30-1991 $\mu \mathrm{g} / \mathrm{L}$ were determined with an accuracy of $100.2-102.2 \%$ and a precision of $4.1-6.4 \%$.

\section{Safety analysis}

Safety assessments included adverse events (AEs), clinical laboratory tests (clinical chemistry, hematology, clotting status, thyroid tests, virology, alcohol breath test, and urinalysis), physical examination, 12-lead electrocardiogram, and measurement of vital signs.

The incidence of treatment-emergent AEs (TEAEs) and drug-related AEs were summarized by treatment using the Medical Dictionary for Regulatory Activities (MedDRA) terms. AEs were considered to be treatment emergent if they had started or worsened after the first application of the study medication and for up to 30 days after the end of treatment with the study medication.

\section{Statistical analyses}

The safety set was defined as all subjects who received at least one dose of either study medication (sorafenib or levothyroxine). The pharmacokinetic analysis set (used for evaluation of the effect of levothyroxine on the pharmacokinetics of sorafenib) was defined as subjects with valid pharmacokinetic profiles of sorafenib in both period 1 (sorafenib alone) and period 2 (sorafenib plus levothyroxine). The pharmacodynamic population was defined as all subjects with valid baseline and at least one valid post baseline thyroid function data point (TSH and total and free T3 and T4). Approximately 25 eligible subjects were planned to enter treatment, with the expectation of approximately 20 evaluable subjects.

Statistical evaluation was performed using the software package SAS v9.2 (SAS Institute, Inc., Cary, NC). Quantitative pharmacodynamic and laboratory safety data were described by summary statistics (arithmetic mean, $S D$, 
median, minimum, and maximum). Summary statistics were provided for both the original data and the change versus baseline whenever appropriate. For pharmacodynamic variables, the baseline of pharmacodynamic parameters was defined as the last pre-dose measurements performed before the first administration of sorafenib in period 2. Qualitative data, such as $\mathrm{AE}$ data, were summarized by frequency.

The plasma concentration versus time profiles of sorafenib and M-2 were summarized separately by treatment. The geometric mean and coefficient of variation were calculated for the concentrations at each of the sampling time points. Means at any time were calculated only if at least two-thirds of the individual data were measured and were above the LLOQ. For the calculation of the mean value, a data point below the LLOQ was substituted by one half of this limit.

Pharmacokinetic parameters ( $t_{\max }$ and $t_{\text {last }}$ excluded) and metabolite ratios were summarized by treatment using the aforementioned statistics. $t_{\max }$ was described using minimum, maximum, and median as well as frequency counts. The pharmacokinetic parameters related to $\mathrm{AUC}_{0 \text {-inf }}$ and $C_{\max }$ of sorafenib and $\mathrm{M}-2$ were analyzed assuming $\log$ normally distributed data using analysis of variance (ANOVA) with treatment as a fixed effect and subject as a random effect. Point estimates (least squares [LS] means), 90\% confidence intervals (CIs), and 95\% prediction intervals for the ratio sorafenib plus levothyroxine (period 2)/sorafenib alone (period 1) were calculated on a logarithmic scale using the intra-individual standard deviation of the ANOVA and transformed back to original scale. Standard safety and clinical laboratory tests were summarized using MedDRA terms.

\section{Results}

\section{Subject disposition and characteristics}

Overall, 51 subjects were enrolled in the study and underwent screening at a single site in Germany. Twenty-two subjects did not fulfill inclusion/exclusion criteria, and four subjects withdrew from the study by consent before initiation of treatment. Thus, 25 subjects entered treatment and completed the study. Of those, one subject did not receive the last dose of levothyroxine but provided all pharmacokinetic samples and completed all visits. All 25 subjects who received study medication were evaluable for safety, pharmacodynamic, and pharmacokinetic analysis sets.

All 25 male subjects were white, with a mean (range) age of $37.3(26-45)$ years, mean $(S D)$ weight of $84.9(11.7) \mathrm{kg}$, height of $182(7.51) \mathrm{cm}$, and BMI of $25.5(2.51) \mathrm{kg} / \mathrm{m}^{2}$. All subjects had normal thyroid function at baseline and were negative for hepatitis B surface antigen, hepatitis $\mathrm{C}$ virus antibodies, and HIV-1 and -2 antibodies.

\section{Pharmacodynamic analysis}

Thyroid tests confirmed that T4, T3, and TSH levels were within reference ranges for all 25 subjects at screening, through period 1, and before levothyroxine administration on day 1 of period 2 (Fig. 2).

Following the daily administration of levothyroxine $300 \mu \mathrm{g}$, free T4 and T3 levels gradually increased, whereas total T4 and T3 had a tendency to increase but remained within the reference ranges, although distributed around the upper normal limits (Fig. 2A and B). Arithmetic mean (SD) values of free T3 and T4 were $4.24(0.66) \mathrm{pg} / \mathrm{mL}$ and 1.77 (0.33) $\mathrm{ng} / \mathrm{dL}$, respectively, by day 11 of period 2 (i.e., after 10 days of levothyroxine $300 \mu \mathrm{g}$ administration). Free $\mathrm{T} 3$ and T4 reached peak values by day 15 of period 2, with an arithmetic mean $(S D)$ of $4.83(1.04) \mathrm{pg} / \mathrm{mL}$ and $1.91(0.30)$ $\mathrm{ng} / \mathrm{dL}$, respectively. Free T3 and T4 levels returned to normal levels for all 25 subjects when assessed at the follow-up visit (Fig. 2A and B).

Corresponding to the increase in free $\mathrm{T} 4$ and $\mathrm{T} 3$, oral doses of levothyroxine $300 \mu$ g q.d. for 14 days successfully induced subclinical thyrotoxicosis, resulting in a steady decrease in TSH (Fig. 2C). By day 11 of period 2, the day when sorafenib was co-administered with levothyroxine, the mean $(S D)$ TSH level had attained full suppression of $0.032(0.027) \mathrm{mIU} / \mathrm{L}$ (about 0.09-fold the lower limit of normal), with the lowest mean $(S D)$ level observed on the last day of levothyroxine dosing $(0.020$ [0.013] $\mathrm{mIU} / \mathrm{L})$. After the last dose of levothyroxine was given, all TSH values returned to normal levels when assessed at the safety follow-up visit (Fig. 2C).

Although levothyroxine treatment induced subclinical biochemical thyrotoxicosis, no clinically relevant signs of hyperthyroidism, such as alterations in heart rate and blood pressure, were observed. Mean $(S D)$ changes from baseline in heart rate in period 2 ranged from -5.8 (10.8) bpm to 5.5 (10.0) bpm. Mean $(S D)$ changes from baseline in systolic blood pressure and diastolic blood pressure ranged from $-5.0(9.4) \mathrm{mmHg}$ to $0.5(9.0) \mathrm{mmHg}$ and -1.6 (7.6) $\mathrm{mmHg}$ to $2.4(6.2) \mathrm{mmHg}$, respectively. No clinically significant changes were observed before or after administration of levothyroxine, regardless of free $\mathrm{T} 4$ and $\mathrm{T} 3$ levels on respective days.

\section{Pharmacokinetic analysis}

Pharmacokinetic parameters are summarized by treatment for sorafenib in Table 1 and Figure 3 and for metabolite M-2 in Table 2 and Figure 4. The pharmacokinetic parameters of sorafenib and its major metabolite M-2 (generated by CYP3A4) were unaffected by co-administration of levothyroxine. Sorafenib pharmacokinetic profiles exhibited multiple peaks (consistent with the known pharmacokinetic profile relevant to enterohepatic circulation of sorafenib), and in some subjects, the second peak was higher than the first peak, resulting in high interindividual variability of $t_{\max }$. Similarly, the plasma concentration versus time profiles of metabolite M-2 also exhibited multiple peaks.

A pairwise comparison of the individual values for sorafenib $\mathrm{AUC}_{0-\text { tlast }}$ following administration of sorafenib alone versus sorafenib plus levothyroxine demonstrated that for the majority of subjects, exposure was slightly lower when levothyroxine and sorafenib were co-administered (period 2) compared to sorafenib alone (period 1). The maximum decrease in exposure observed for an individual subject was approximately $50 \%$. Although a few subjects showed an increase in sorafenib exposure in period 2, no subject was considered to be an outlier because all measured exposures were consistent with historic data for a sorafenib $400 \mathrm{mg}$ single dose in healthy volunteers (data on file; Bayer HealthCare Pharmaceuticals) (21).

Similarly, a pairwise comparison of the individual values for $\mathrm{AUC}_{0-\text { tlast }}$ of metabolite $\mathrm{M}-2$ for sorafenib alone 
FIG. 2. Mean $(S D)$ thyroid test values over time for (A) free T4 and total T4, (B) free T3 and total T3, and (C) TSH (mIU/L). M, minutes; P1, period 1, sorafenib; $\mathrm{P} 2$, period 2 , sorafenib plus levothyroxine. The time point reference is the first dose of sorafenib in the period.

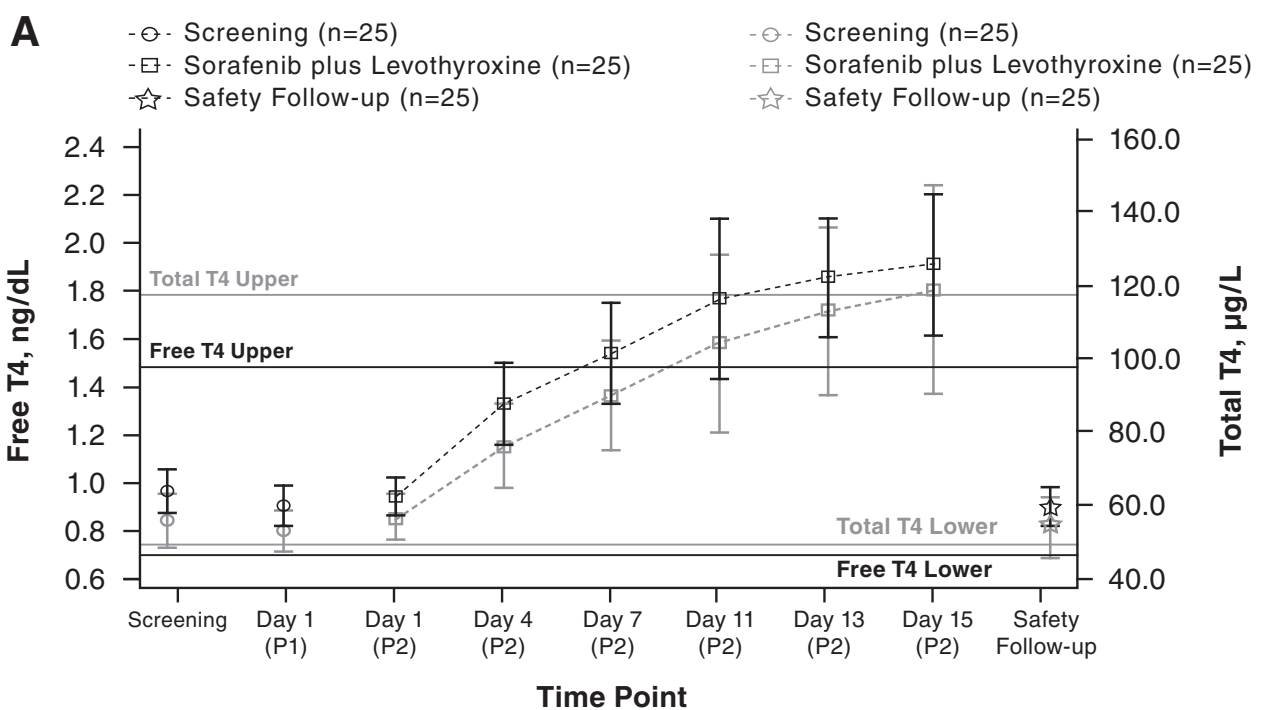

B

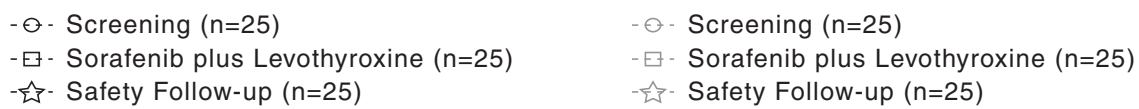

- $E$ - Sorafenib plus Levothyroxine $(n=25)$

- - Safety Follow-up $(n=25)$

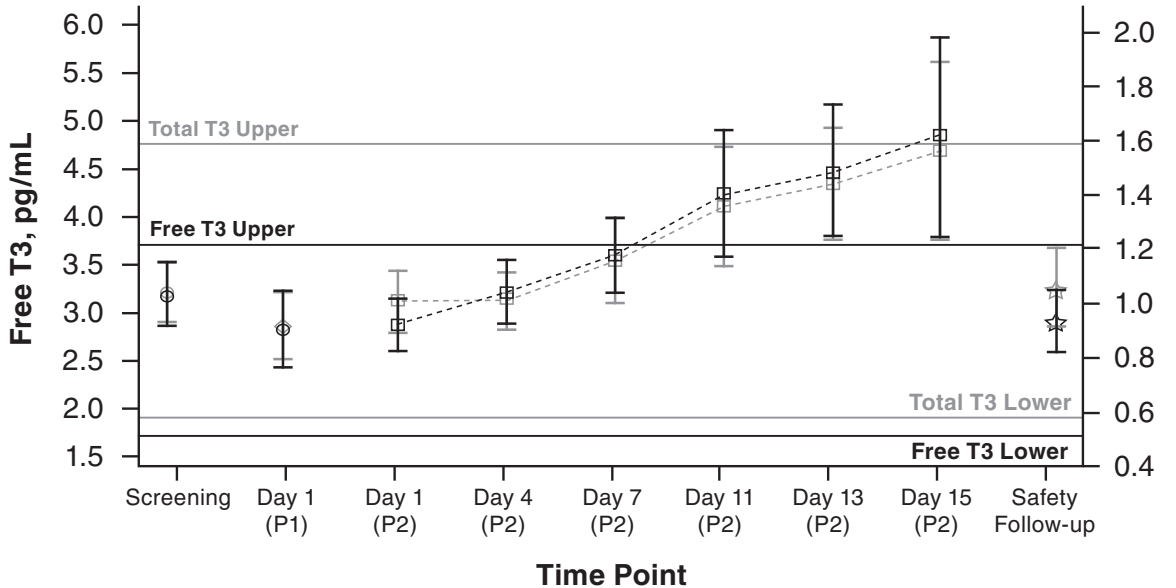

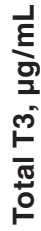

- - . Sorafenib plus Levothyroxine $(n=25)$ -

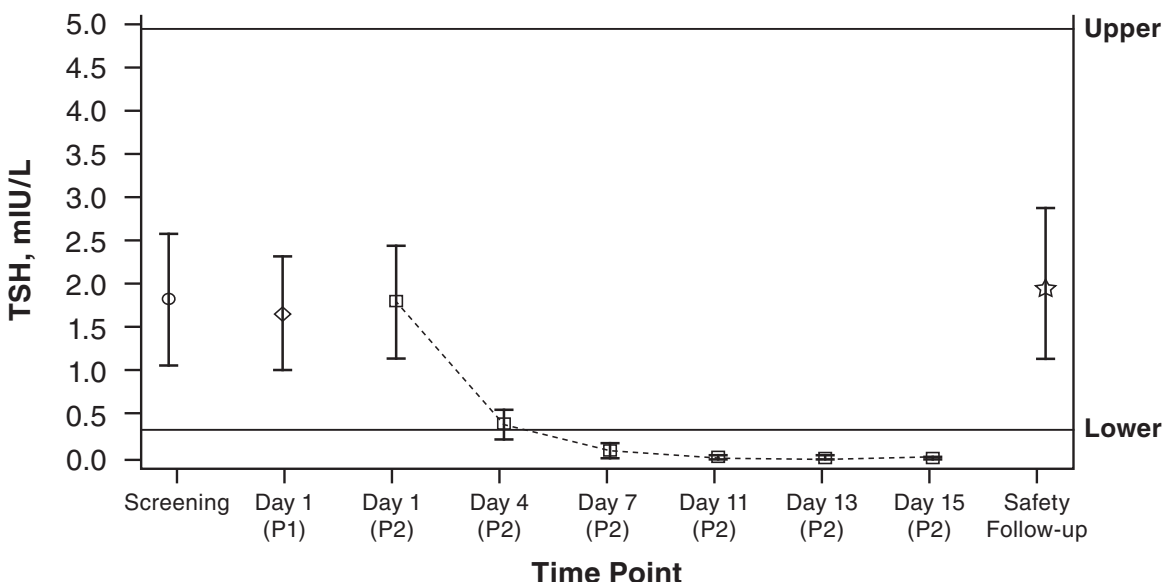


Table 1. Pharmacokinetic Parameters of Sorafenib in Plasma Following Single Oral Administration of 400 mg of Sorafenib Alone (Day 1) and With $300 \mu \mathrm{g}$ of Levothyroxine (Day 11)

\begin{tabular}{lcc}
\hline & & Mean ${ }_{\text {ged }} / C V \%($ range $)$ \\
\cline { 2 - 3 } Parameter & Sorafenib $(\mathrm{n}=25)$ & Sorafenib + levothyroxine $(\mathrm{n}=25)$ \\
\hline $\mathrm{AUC}_{0-\mathrm{inf}}, \mathrm{mg} \cdot \mathrm{h} / \mathrm{L}$ & $68.1 / 68.2(19.3-239)$ & $64.3 / 66.3(19.6-295)$ \\
$\mathrm{AUC}_{0-\mathrm{tlast}}, \mathrm{mg} \cdot \mathrm{h} / \mathrm{L}$ & $62.8 / 68.3(17.6-228)$ & $58.5 / 67.1(16.8-261)$ \\
$\mathrm{AUC}$ & $6-96, \mathrm{mg} \cdot \mathrm{h} / \mathrm{L}$ & $58.5 / 67.1(16.8-262)$ \\
$C_{\max }, \mathrm{mg} / \mathrm{L}$ & $2.09 / 68.3(17.6-227)$ & $1.78 / 63.9(0.589-6.04)$ \\
$t_{\max }, \mathrm{h}^{\mathrm{a}}$ & $4.00(2.98-16.0)$ & $4.02(1.98-36.0)$ \\
$t_{1 / 2}, \mathrm{~h}$ & $24.0 / 25.3(16.2-43.2)$ & $25.7 / 21.0(17.6-43.0)$ \\
\hline
\end{tabular}

${ }^{\mathrm{a}}$ Median (range).

$\mathrm{AUC}_{0-\text { inf }}$, area under the concentration versus time curve from time zero to infinity after single (first) dose; $\mathrm{AUC}_{0-96}$, area under the plasma concentration versus time curve from time 0 to 96 hours; $\mathrm{AUC}_{0-\text { tlast}}$, $\mathrm{AUC}$ from time 0 to the last data point greater than the lower limit of quantification; $C_{\mathrm{max}}$, maximum observed drug concentration in measured matrix after single dose administration; CV, coefficient of variation; mean $_{\mathrm{geo}}$, geometric mean; $t_{1 / 2}$, half-life associated with the terminal slope; $t_{\max }$, time to reach $C_{\max }$.

versus co-administration of levothyroxine with sorafenib demonstrated that a majority of subjects had slightly lower metabolite M-2 exposure when sorafenib was coadministered with levothyroxine. A few subjects also had increased metabolite M-2 exposure in period 2, with maximum effects similar to those observed for sorafenib alone.

For selected pharmacokinetic parameters, point estimates (LS means) and 90\% CIs for the ratio of sorafenib plus le- vothyroxine (period 2) to sorafenib alone (period 1) were calculated to evaluate the effect of hyperthyroidism and levothyroxine co-administration on the pharmacokinetics of sorafenib (Table 3). Point estimates for $\mathrm{AUC}_{0-\mathrm{inf}}, \mathrm{AUC}_{0-\text { tlast }}$, $\mathrm{AUC}_{0-96}$, and $C_{\max }$ of sorafenib ratios comparing period 2 (sorafenib plus levothyroxine) to period 1 (sorafenib alone) were between 0.85 and 0.94 . Respective point estimates for $\mathrm{AUC}_{0-\mathrm{inf}}, \mathrm{AUC}_{0-\text { tlast }}$, and $C_{\text {max }}$ of metabolite $\mathrm{M}-2$ ratios were

A

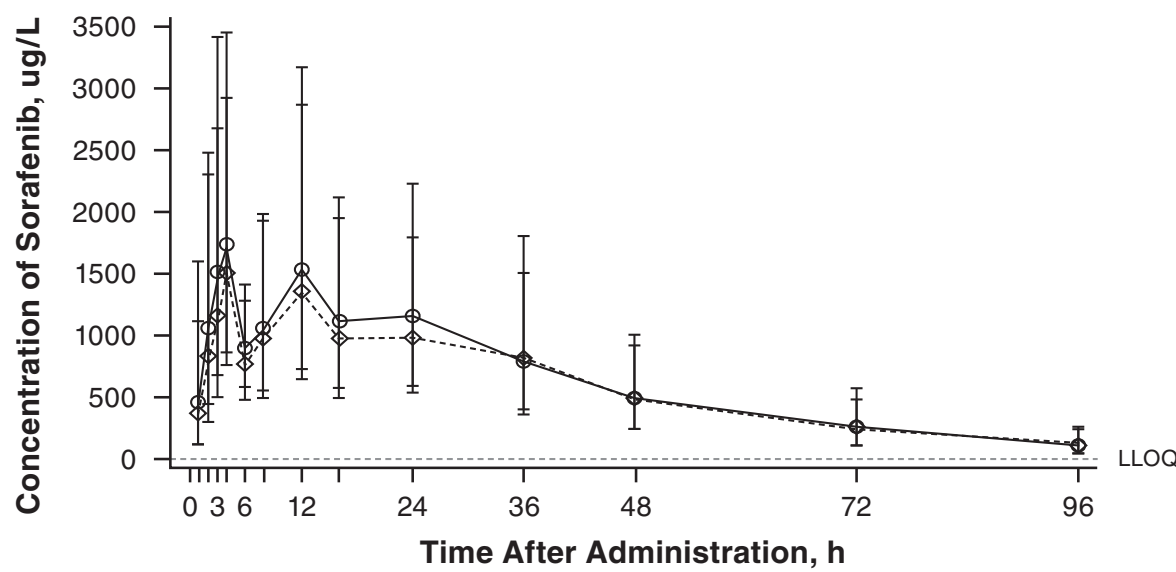

B

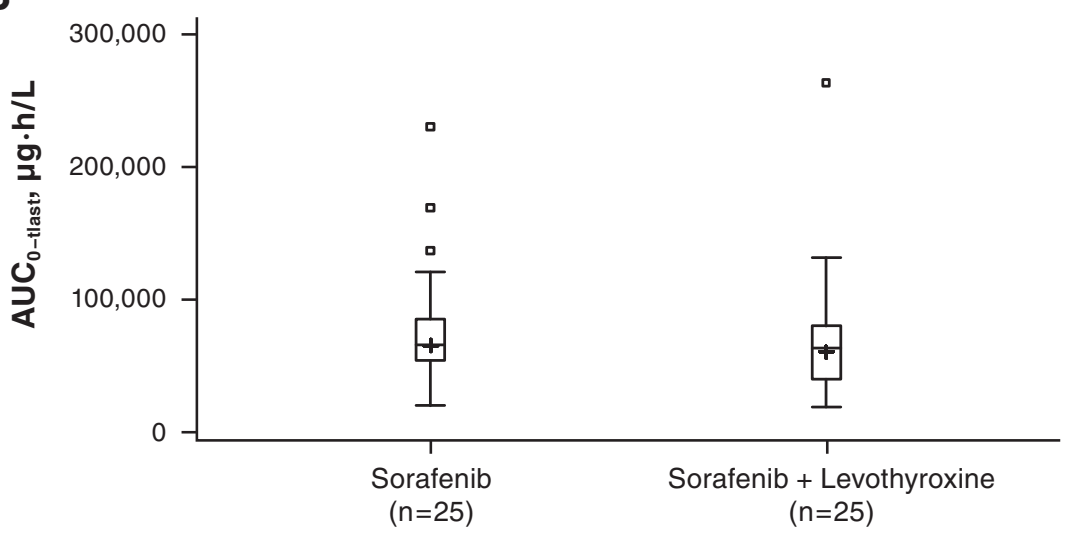

FIG. 3. Geometric mean (Geom. $S D)$ plasma concentration-time profiles of sorafenib $(\mu \mathrm{g} / \mathrm{L})$ following single oral administration of sorafenib $400 \mathrm{mg}$ alone or coadministered with levothyroxine $300 \mu \mathrm{g}$ shown as (A) linear scale and $(\mathbf{B})$ box plot for $\mathrm{AUC}_{(0-\text { tlast })}$ of sorafenib in plasma by treatment. LLOQ, lower limit of quantification.

Treatment 
Table 2. Pharmacokinetic Parameters of Metabolite M-2 in Plasma Following Single Oral Administration of 400 mg of Sorafenib Alone (Day 1) and With 300 mg of LeVothyroxine (Day 11)

\begin{tabular}{lcc}
\hline & & Mean $_{\text {ged }} /$ CV\% (range) \\
\cline { 2 - 3 } Parameter & Sorafenib $(\mathrm{n}=25)$ & Sorafenib + levothyroxine $(\mathrm{n}=25)$ \\
\hline $\mathrm{AUC}_{0-\mathrm{inf}}, \mathrm{mg} \cdot \mathrm{h} / \mathrm{L}$ & $16.3 / 69.3(4.10-55.4)^{\mathrm{a}}$ & $12.8 / 87.2(4.31-84.3)^{\mathrm{b}}$ \\
$\mathrm{AUC}$ & & $9.47 / 137(6.76-74.7)$ \\
$C_{\max }, \mathrm{mg} / \mathrm{L}$ & $11.2 / 127(1.23-52.2)$ & $0.348 / 110(0.0556-1.75)$ \\
$t_{\max }, \mathrm{h}^{\mathrm{c}}$ & $0.427 / 111(0.0491-1.49)$ & $4.00(1.98-16.0)$ \\
$t_{1 / 2}, \mathrm{~h}$ & $4.00(2.98-16.0)$ & $25.6 / 21.7(16.6-40.9)^{\mathrm{b}}$ \\
\hline
\end{tabular}

In case of two identical $C_{\max }$ values, the first $t_{\max }$ was used.

${ }^{\mathrm{a}} n=22$ for sorafenib.

${ }^{b} n=23$ for sorafenib + levothyroxine.

${ }^{\mathrm{c}}$ Median (range).

between 0.82 and 0.85 . The $90 \%$ CIs for the ratios of all parameters of sorafenib and metabolite M-2 included unity, indicating that exposure and $C_{\max }$ of sorafenib and its metabolite M-2 were not influenced by the multiple-dosing regimen of levothyroxine. Consequently, these results demonstrate the absence of a significant effect of levothyroxine on the pharmacokinetics and exposure of sorafenib or its metabolite M-2.

\section{Safety}

A single oral dose of sorafenib $400 \mathrm{mg}$ administered alone or in combination with levothyroxine $300 \mu \mathrm{g}$ was generally well tolerated. Overall, $96.0 \%(n=24)$ of subjects (period 1, $52 \%[n=13]$; period $2,88 \%[n=22])$ who had received treatment experienced $\geq 1$ TEAE. The most frequently reported TEAEs were headache (48\%: one subject in period 1
FIG. 4. Geometric mean (Geom. $S D)$ plasma concentration-time profiles of metabolite $\mathrm{M}-2(\mu \mathrm{g} / \mathrm{L})$ following single oral administration of sorafenib $400 \mathrm{mg}$ alone or coadministered with levothyroxine $300 \mu \mathrm{g}$ shown as (A) linear scale and $(\mathbf{B})$ box plot for $\mathrm{AUC}_{(0-\text { tlast })}$ of M-2 in plasma by treatment; $N=25$.

\section{A}
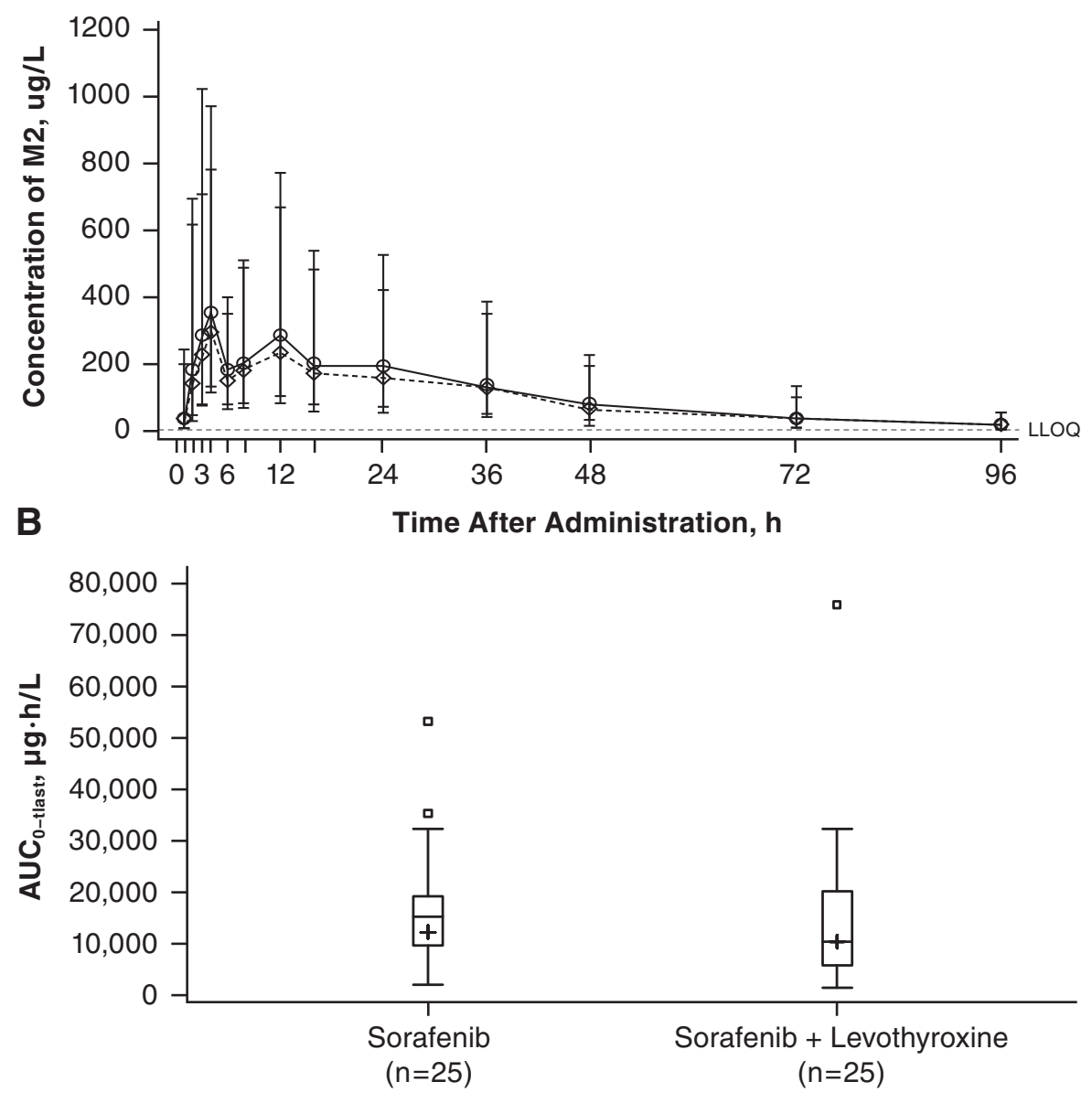

Treatment 
Table 3. Point Estimates, $90 \%$ Cis, and 95\% Prediction Intervals of the Ratio of Sorafenib Plus Levothyroxine (Period 2)/Sorafenib Alone (Period 1) for Selected Pharmacokinetic Parameters of Sorafenib and Metabolite M-2

\begin{tabular}{lccccc}
\hline & \multicolumn{2}{c}{ Sorafenib $(\mathrm{n}=25)$} & & \multicolumn{2}{c}{ Metabolite M-2 (n=25) } \\
\cline { 2 - 3 } & LS mean of ratio $(90 \%$ CI) & $95 \%$ Prediction interval & & LS mean of ratio (90\% CI) & 95\% Prediction interval \\
\hline AUC $_{0-\text { inf }}$ & $0.9435(0.7869-1.1313)$ & $0.3090-2.8811$ & & $0.8210(0.6123-1.1008)$ & $0.1500-4.4928^{\mathrm{a}}$ \\
AUC $_{0-\text { tlast }}$ & $0.9317(0.7733-1.1224)$ & $0.2963-2.9292$ & & $0.8462(0.6387-1.1212)$ & $0.1499-4.7772$ \\
AUC $_{0-96}$ & $0.9325(0.7740-1.1234)$ & $0.2966-2.9322$ & & NA & NA \\
$C_{\text {max }}$ & $0.8529(0.7076-1.0281)$ & $0.2703-2.6915$ & & $0.8151(0.6270-1.0596)$ & $0.1624-4.0922$ \\
\hline
\end{tabular}

${ }^{\mathrm{a}} n=22$.

LS, least squares; NA, not applicable.

and 12 subjects in period 2), fatigue (32.0\%: three subjects and six subjects, respectively), and increased blood creatine kinase (24\%: three subjects and four subjects, respectively). Most AEs were mild $(88.0 \% ; n=22)$ or moderate $(24.0 \%$; $n=5)$ in intensity. No TEAEs were considered to be severe.

Sorafenib-related TEAEs were reported in $28 \%(n=7)$ of subjects in period 1 and in $40 \%(n=10)$ of subjects in period 2 (56\% overall for periods 1 and $2 ; n=14$ ). Levothyroxinerelated TEAEs were experienced by $72.0 \%(n=18)$ of subjects in period 2; subjects were not exposed to levothyroxine in period 1. There were no deaths, serious AEs, or discontinuations due to AEs in subjects treated with sorafenib and/or levothyroxine.

The most commonly reported sorafenib-related TEAEs were headache $(28 \%)$, fatigue $(16 \%)$, and increased amylase $(8 \%)$ and lipase $(8 \%)$. The most commonly reported levothyroxine-related TEAEs were headache $(32 \%)$, increased alanine aminotransferase (ALT; 16\%), fatigue (12\%), increased glutamate dehydrogenase (GLDH; 12\%), and nervousness $(12 \%)$.

No clinically significant changes in laboratory values due to treatment were observed. Laboratory values that were more than twice the upper limit of normal included Creactive protein $(n=2)$, aspartate aminotransferase $(n=1)$, ALT $(n=2)$, GLDH $(n=3)$, creatine phosphokinase $(n=6)$, and triglycerides $(n=1)$. However, these elevations were not considered to be of medical concern. Sorafenib, levothyroxine, and the co-administration of both agents did not influence the overall pattern of vital signs, including heart rate and blood pressure, over time. There were no clinically significant changes in electrocardiogram parameters due to study medications.

\section{Discussion}

This study investigated the effect of levothyroxineinduced subclinical thyrotoxicosis on the pharmacokinetics of sorafenib. In healthy male subjects with normal thyroid function, levothyroxine $300 \mu \mathrm{g}$ q.d. for 14 days successfully induced subclinical thyrotoxicosis, as indicated by full suppression of TSH and an accompanying elevation in free T3 and T4 levels, albeit total T3 and T4 still within the reference range. A trend toward increasing levels of T3 and T4 over time was observed, corresponding with levothyroxine daily dosing. A full normalization was achieved after stopping levothyroxine. Importantly, co-administration of levothyroxine with sorafenib had no influence on the pharmacoki- netics of sorafenib and its metabolite M-2. In addition, no new safety concerns emerged; sorafenib administered without and with levothyroxine was generally well tolerated.

The current analysis was conducted to explore the possible mechanism for the increased sorafenib exposure in patients with DTC compared to HCC and RCC (13). The majority of patients in the pivotal Phase 3 DTC study had received levothyroxine, whereas only a few patients in the prior HCC or RCC studies had been co-administered this agent. Thus, it was hypothesized that levothyroxine's known wide drug interaction spectrum, CYP3A4 inhibition, and capacity to increase the exposure of numerous drugs, including some tyrosine kinase inhibitors (15), might be responsible for the increased sorafenib exposure observed in the DTC study. The present results demonstrate that neither the levothyroxineinduced subclinical thyrotoxicosis nor drug-drug interaction when levothyroxine was given concomitantly with sorafenib significantly affected the pharmacokinetic profile of sorafenib, including sorafenib exposure. Thus, it is unlikely that there will be drug-drug interaction concerns in the clinic when sorafenib is administered concomitantly to levothyroxinetreated DTC patients.

The reason for increased sorafenib exposure in patients with DTC is currently unknown. It is unlikely that decreased sorafenib metabolism by CYP3A4 would result in increased exposure because metabolite M-2 levels have been shown to remain high in DTC patients in DECISION, nor does there seem to be a relationship between free/total T3 and T4 levels and sorafenib/M-2 levels in DECISION (data on file; Bayer HealthCare Pharmaceuticals). Similarly, a potential inhibitory effect of T3/T4 on UGT1A9 glucuronidation of sorafenib is not supported by previous in vitro study data (data on file; Bayer HealthCare Pharmaceuticals). Although unidentified factors may contribute to the observed increase in sorafenib exposure in DECISION, the apparent increase might be simply a consequence of the high inter-subject pharmacokinetic variability inherent to sorafenib. Importantly, although the mechanism of action for the increase in sorafenib exposure in the DTC study still requires elucidation, Bastholt et al. have shown that there is no clinically relevant correlation between sorafenib exposure $\left(\mathrm{AUC}_{0-\mathrm{inf}}\right)$ and the incidence or severity of AEs (13). Consequently, the increased sorafenib exposure observed in the DTC population may not be clinically meaningful.

The safety results in the current study showed that the high dose of levothyroxine $(300 \mu \mathrm{g})$ was well tolerated in healthy subjects for 14 days of treatment. The majority of AEs were 
mild in intensity, with headache being the most common drug-related AE for both agents. No serious AEs were reported, and no subject discontinued treatment because of an AE. There were no clinically relevant changes in electrocardiograms, blood pressure readings, or heart rate after 14 days of $300 \mu \mathrm{g}$ q.d. levothyroxine treatment. Clinical laboratory assessments also showed no relevant changes of clinical significance associated with the regimen.

There are several limitations to the current study. One limitation is the relatively short duration of exposure to levothyroxine compared to the treatment duration typically experienced by patients with DTC. Thus, the possibility of a cumulative effect associated with longer exposure times to levothyroxine in patients with DTC in combination with long-term sorafenib dosing cannot be eliminated. Although published data support the safety of dosing healthy subjects with a high dose of levothyroxine for up to 14 days to achieve thyrotoxic levels (22), more prolonged dosing of healthy volunteers was not considered feasible. Furthermore, this study was not designed to investigate potential effects of sorafenib on levothyroxine pharmacokinetics, as our specific concern focused on the apparent alteration of sorafenib pharmacokinetics in the DECISION trial. Thus, the study only included single-dose pharmacokinetic evaluation of sorafenib because it was not justified to expose healthy subjects to multiple doses of sorafenib. Nevertheless, since multiple-dose pharmacokinetics of sorafenib and its metabolites are consistent with single-dose results, the drug interaction effects of levothyroxine on single-dose sorafenib can be extrapolated to the multiple-dose situation. However, single-dose levothyroxine pharmacokinetics without sorafenib were not measured in this study, so no conclusion on the influence of sorafenib on levothyroxine can be deduced.

Another limitation of this study is that the subjects in this analysis were all healthy volunteers. It is unknown whether there may be an undetermined factor or factors inherent to DTC patients that could result in increased sorafenib exposure. Although there are clear advantages to performing the study in DTC patients, this approach was not feasible, as all patients should have received levothyroxine without interruption as part of clinical standard of care to achieve a full suppression of TSH to lower the risk of cancer recurrence (10). Although subclinical thyrotoxicosis was induced by continuous oral intake of high doses of levothyroxine for 14 days without major safety concerns, the limitations of this model of thyrotoxicosis are not clear (23).

In this Phase 1 open-label study in healthy men, subclinical thyrotoxicosis was successfully induced by continuous oral daily administration of $300 \mu \mathrm{g}$ of levothyroxine and did result in a significant increase in free T3 and T4 levels, albeit still within the reference ranges, and full suppression of $\mathrm{TSH}$, without the development of clinically significant symptoms. Co-administration of levothyroxine with sorafenib had no influence on the pharmacokinetics of sorafenib and its metabolite M-2. The safety profile of sorafenib when co-administered with levothyroxine was consistent with its known safety profile when given alone. High-dose levothyroxine $(300 \mu \mathrm{g})$ was well tolerated with continuous daily dosing for 14 days. These findings suggest that there are no concerns when coadministering levothyroxine with sorafenib in patients with DTC. The mechanism of action for the increased sorafenib exposure in patients with DTC is unlikely to be related to the levothyroxine treatment indicated for patients with DTC. Mechanisms regarding the relatively high exposure in patients with DTC compared to other types of cancer remain elusive.

\section{Acknowledgments}

Scientific writing support for development of this manuscript was provided by Alan J. Klopp, PhD, CMPP, and Lisa M. Klumpp Callan, PhD, at C4 MedSolutions, LLC (Yardley, PA), a CHC Group company, and funded by Bayer HealthCare Pharmaceuticals. Data from this study were previously presented at the 86th Annual Meeting of the American Thyroid Association; September 21-25, 2016, Denver, CO.

\section{Author Disclosure Statement}

The study was conducted on behalf of the sponsor, Bayer HealthCare Pharmaceuticals. F.H., A.A.-H., E.H., J.L., R.L., and C.P. are employed by Bayer HealthCare Pharmaceuticals. C.P. owns stock in Bayer. M.B. is employed by CRS Clinical Research Services Berlin GmbH.

\section{References}

1. National Cancer Institute. A snapshot of thyroid cancerincidence and mortality. Available at: www.cancer.gov/ research/progress/snapshots/thyroid (accessed April 1, 2016).

2. National Cancer Institute Surveillance, Epidemiology, and End Results Program. SEER Stat fact sheets: thyroid cancer. Available at: http://seer.cancer.gov/statfacts/html/thyro .html (accessed April 1, 2016).

3. ITOG 2016 International Thyroid Oncology Group: poorly differentiated thyroid cancer. Available at: www.itog.org/ poorly-differentiated-thyroid-cancer (accessed July 21, 2016).

4. Nikiforov YE 2011 Molecular diagnostics of thyroid tumors. Arch Pathol Lab Med 135:569-577.

5. ThyCa: Thyroid Cancer Survivors' Association, Inc. Facts about thyroid cancer. Available at: www.thyca.org/about/ thyroid-cancer-facts/ (accessed April 1, 2016).

6. Wilhelm S, Carter C, Lynch M, Lowinger T, Dumas J, Smith RA, Schwartz B, Simantov R, Kelley S 2006 Discovery and development of sorafenib: a multikinase inhibitor for treating cancer. Nat Rev Drug Discov 5:835-844.

7. Wilhelm SM, Adnane L, Newell P, Villanueva A, Llovet JM, Lynch M 2008 Preclinical overview of sorafenib, a multikinase inhibitor that targets both Raf and VEGF and PDGF receptor tyrosine kinase signaling. Mol Cancer Ther 7:3129-3140.

8. Duntas LH, Bernardini R 2010 Sorafenib: rays of hope in thyroid cancer. Thyroid 20:1351-1358.

9. Nexavar (sorafenib) prescribing information. Bayer HealthCare Pharmaceuticals, Inc., Whippany, NJ, revised 2015. Available at: http://bayer.c4share.net/NEX15715/1000\%20 Manuscripts/NEX15715.1009\%20Study\%2017436\%20 Healthy\%20Volunteers/Resources/Nexavar_PI.pdf (accessed August 10, 2017).

10. Haugen BR, Alexander EK, Bible KC, Doherty GM, Mandel SJ, Nikiforov YE, Pacini F, Randolph GW, Sawka AM, Schlumberger M, Schuff KG, Sherman SI, Sosa JA, Steward DL, Tuttle RM, Wartofsky L 20162015 American Thyroid Association management guidelines for adult patients with thyroid nodules and differentiated thyroid cancer: the American Thyroid Association Guidelines Task Force on Thyroid Nodules and Differentiated Thyroid Cancer. Thyroid 26:1-133. 
11. Brose MS, Nutting CM, Jarzab B, Elisei R, Siena S, Bastholt L, de la Fouchardiere C, Pacini F, Paschke R, Shong YK, Sherman SI, Smit JW, Chung J, Kappeler C, Pena C, Molnar I, Schlumberger MJ; DECISION Investigators 2014 Sorafenib in radioactive iodine-refractory, locally advanced or metastatic differentiated thyroid cancer: a randomised, double-blind, phase 3 trial. Lancet 384:319-328.

12. Savvides P, Nagaiah G, Lavertu P, Fu P, Wright JJ, Chapman R, Wasman J, Dowlati A, Remick SC 2013 Phase II trial of sorafenib in patients with advanced anaplastic carcinoma of the thyroid. Thyroid 23:600-604.

13. Bastholt L, Brose MS, Jarzab B, Schlumberger M, Siena S, De La Fouchardiere C, Paschke R, Deshpande HA, Shi Y, Elisei R, Gao M, Li L, Prins K, Walker H, Mitchell DY, Lettieri JT, Molnar I, Kappeler C, Pena CE 2014 Population PK modeling and exposure-response analyses of sorafenib in patients with radioactive iodine-refractory differentiated thyroid cancer (RAI-rDTC) in the Phase III DECISION trial. J Clin Oncol 32:5s.

14. National Comprehensive Cancer Network. NCCN Clinical Practice Guidelines in Oncology (NCCN Guidelines ${ }^{\circledR}$ ): thyroid Carcinoma, version 2.2015. Available at: www .nccn.org/professionals/physician_gls/pdf/thyroid.pdf (accessed April 1, 2016).

15. Haouala A, Widmer N, Duchosal MA, Montemurro M, Buclin T, Decosterd LA 2011 Drug interactions with the tyrosine kinase inhibitors imatinib, dasatinib, and nilotinib. Blood 117:e75-87.

16. Zhao H-D, Bao G-Q, He X-L, Wu T, Wang C-G, Wang S-Z, Zang L, Lu J-G, Du X-L 2012 Strong inhibition of UDP-glucuronosyltransferase (UGT) 1A1 by levothyroxine indicates the potential UGT-inhibition based adverse effect of levothyroxine. Lat Am J Pharm 31:761-763.

17. Takahashi N, Inui $\mathrm{N}$, Morita $\mathrm{H}$, Takeuchi $\mathrm{K}$, Uchida $\mathrm{S}$, Watanabe H, Nakamura H 2010 Effect of thyroid hormone on the activity of CYP3A enzyme in humans. J Clin Pharmacol 50:88-93.
18. Lathia C, Lettieri J, Cihon F, Gallentine M, Radtke M, Sundaresan P 2006 Lack of effect of ketoconazolemediated CYP3A inhibition on sorafenib clinical pharmacokinetics. Cancer Chemother Pharmacol 57:685-692.

19. U.S. Department of Health and Human Services, Food and Drug Administration, Center for Drug Evaluation and Research (CDER), Center for Veterinary Medicine (CVM). Guidance for industry: bioanalytical method validation. Available at: www.fda.gov/downloads/Drugs/ GuidanceComplianceRegulatoryInformation/Guidances/ ucm070107.pdf (accessed April 1, 2016).

20. European Medicines Agency, Committee for Medicinal Products for Human Use (CHMP). Guideline on Bioanalytical Method Validation. Available at: www.ema.europa. eu/docs/en_GB/document_library/Scientific_guideline/2011/ 08/WC500109686.pdf (accessed April 1, 2016).

21. Lettieri J, Lathia C, Rotolo C 2011 Effect of neomycin on the pharmacokinetics of sorafenib. Clin Pharmacol Ther 89:S54.

22. Piolino V, Acheson KJ, Muller MJ, Jeanpretre N, Burger AG, Jequier E 1990 Thermogenic effect of thyroid hormones: interactions with epinephrine and insulin. Am J Physiol 259:E305-311.

23. Johannsen DL, Galgani JE, Johannsen NM, Zhang Z, Covington JD, Ravussin E 2012 Effect of short-term thyroxine administration on energy metabolism and mitochondrial efficiency in humans. PLoS One 7:e40837.

Address correspondence to: Funan Huang, MD, PhD Bayer HealthCare 100 Bayer Boulevard Whippany, NJ 07981-0915

E-mail: funan.huang@bayer.com 